\title{
CONVERGENCE OF OPTIMAL STRATEGIES UNDER PROPORTIONAL TRANSACTION COSTS
}

\author{
RAFAŁ KUCHARSKI \\ Institute of Mathematics, Polish Academy of Sciences \\ Sniadeckich 8, 00-956 Warszawa, Poland \\ E-mail: kucharski@impan.pl
}

\begin{abstract}
A discrete-time financial market model with finite time horizon and transaction costs is considered, with a sequence of investors whose preferences are described by a convergent sequence of strictly increasing and strictly concave utility functions. Proportional costs are approximated by strictly convex costs. Existence of the optimal consumption-investment strategies is obtained, as well as convergence of the value functions and convergence of subsequences of optimal strategies.
\end{abstract}

Introduction. When we consider the problem of utility maximization, the following natural question arises: do optimal strategies depend continuously on investors' preferences? More precisely: if a sequence of utility functions converges to some utility function, do the respective strategies converge? Recently a number of papers gave an affirmative answer to this question. In continuous time, Jouni and Napp [9] considered a Brownian motion-driven complete market model, and Larsen [8] extended their considerations to a general continuous semi-martingale model, while papers [1] and [7] dealt with discrete time models with finite horizon.

In this paper we are going to modify the model described in [7] by taking into consideration transaction costs. This forces us to model our market as a multidimensional dynamical system, and direct application of methods used in the latter paper is not possible.

We are going to consider a market with discrete time and finite horizon. A finite number of trading assets is available, among which there may be (but doesn't have to be) a riskless one. There is no short selling. Regularity conditions on utility functions we will assume are rather weak: strict concavity and strict monotonicity. Similarly to [11]

2000 Mathematics Subject Classification: Primary 91B28; Secondary 93E20.

Key words and phrases: utility maximization, optimal strategies, transaction costs, strategies convergence, strictly convex costs.

The paper is in final form and no version of it will be published elsewhere. 
and [7], utility functions considered in the paper are defined on the positive axis. It's worth to notice that utility functions may be different in every moment of time.

To begin with strictly convex costs we consider a one-step model and prove existence and uniqueness of optimal strategies, as well as their convergence. With these results and dynamic programming technique we show the same for an arbitrary finite time horizon. In the method we use uniqueness of optimal strategies is crucial. Since we are not able to obtain uniqueness for proportional costs, only partial results are presented: convergence of value functions and existence of convergent subsequence of optimal strategies. Although the main goal of using strictly convex costs is to approximate proportional ones, the results obtained may also be interesting, as strictly convex costs are suitable for modelling liquidity effects, see [2].

The structure of the paper is as follows: in the first section we describe the details of our model of the financial market and introduce cost functions. Then we consider the "one step" case for the market with strictly convex costs and establish useful technical results concerning existence, uniqueness and convergence of optimal strategies on such markets. The general case and the main results for proportional costs are presented in the last section.

We use dynamic programming methods and for these refer the reader to [12]. We also make extensive use of two lemmas, which we present in the Appendix. The first one comes from the celebrated paper of Kabanov and Stricker [5], and the second can be derived e.g. from the results contained in [10], but for completeness we attach an elementary proof.

1. Market model. Assume that on a probability space $\left(\Omega, \mathcal{F},\left(\mathcal{F}_{t}\right)_{0 \leq t \leq T}, P\right)$ with discrete filtration and finite time horizon $T \in \mathbb{N}$ we are given a $d$-dimensional process $\left\{S_{t}=\left(S_{t, 1}, \ldots, S_{t, d}\right), 0 \leq t \leq T\right\}$ with strictly positive coordinates, describing the prices of $d$ available securities, which we will call shares. We assume that $\mathcal{F}_{0}$ is trivial. The dynamics of $S_{t}$ is of the form

$$
\zeta_{t, i}=\frac{S_{t+1, i}}{S_{t, i}}, \quad i=1, \ldots, d,
$$

where $\zeta_{t}=\left(\zeta_{t, 1}, \ldots, \zeta_{t, d}\right)$ is an $\mathcal{F}_{t+1}$-adapted random vector. Throughout the paper the following notation will be used: by $\mathbb{R}^{d}$ we mean $d$-dimensional Euclidean space with the standard inner product $\langle x, y\rangle=\sum_{i=1}^{d} x_{i} y_{i}, x, y \in \mathbb{R}^{d}$ and partial order: $x \leq y \Leftrightarrow x_{i} \leq y_{i}$ for $i=1, \ldots, d$. We write $x<y$ when $x \leq y$ and $x \neq y$. If $x \in \mathbb{R}^{d}$, then $x \cdot \mathbf{1}:=\sum_{i=1}^{d} x_{i}$ denotes the sum of its coordinates. Let $\mathbb{R}_{++}^{d}=[0, \infty)^{d} \backslash\{0\}$ denote the positive orthant in $\mathbb{R}^{d}$,

$$
\Delta_{0}=\left\{\nu=\left(\nu_{1}, \ldots, \nu_{m}\right) \in \mathbb{R}^{d}: \nu_{i} \geq 0, \nu \cdot \mathbf{1} \leq 1\right\},
$$

and $\Delta=\left\{\nu \in \Delta_{0}: \nu \cdot \mathbf{1}=1\right\}$. To simplify the presentation we introduce the mapping $h: \mathbb{R}_{++}^{d} \times \mathbb{R}_{++}^{d} \times \mathbb{R}_{++}^{d} \rightarrow \mathbb{R}_{++}^{d}, h:=\left(h_{1}, \ldots, h_{d}\right)^{\prime}$ where

$$
h_{i}(\pi, \zeta, \theta):=\pi_{i} \zeta_{i}(\theta \cdot \mathbf{1}), \quad i=1, \ldots, d .
$$

For indexing we will often use the set $\overline{\mathbb{N}}=\mathbb{N} \cup\{\infty\}$ of natural numbers with an additional element. 
1.1. Market dynamics. Investors present on the market at every time $t=0, \ldots, T-1$ are allowed to consume a part of their wealth and change their portfolios in order to maximize the expected utility of the consumption and terminal wealth (portfolio value), in a way we are now going to make precise. An investor starts at time $t=0$ with an initial portfolio $\theta=\theta_{0}=\left(\theta_{0,1}, \ldots, \theta_{0, d}\right)$, where $\theta_{t, i}, t=0, \ldots, T, i=1, \ldots, d$, denotes the capital held in the $i$-th security at time $t$ before consumption and transactions. At time $t$ he consumes a part $\alpha_{t}$ of his total wealth $X_{t}=\theta_{t} \cdot \mathbf{1}$ (that is, the amount $\alpha_{t} X_{t}$ ), and chooses a new portfolio $\theta_{t}^{+}=\left(\theta_{0,1}^{+}, \ldots, \theta_{0, d}^{+}\right)$. Decisions about consumption and new portfolio are based on current information, which means that we assume the process $\left(\alpha_{t}, \theta_{t}^{+}\right)$is adapted. Since we do not allow borrowing and short selling, we have $\alpha_{t} \in[0,1]$ and $\theta_{t, i}^{+} \geq 0$ for all $t=0, \ldots, T, i=1, \ldots, d$.

In order to change the portfolio or consume wealth, investor has to sell or buy some shares, and doing that, he bears transaction costs. Namely, changing the portfolio from $\theta_{t}$ to $\theta_{t}^{+}$means diminishing wealth by a cost $c\left(\theta_{t}, \theta^{+}\right)$. These operations lead us to the following equation:

$$
X_{t}=\alpha_{t} X_{t}+c\left(\theta_{t}, \theta_{t}^{+}\right)+\theta_{t}^{+} \cdot \mathbf{1}
$$

describing the distribution of the investor's wealth among consumption, transaction costs and new portfolio at time $t$. If we denote by

$$
\pi_{t, i}=\frac{\theta_{t, i}^{+}}{X_{t}}
$$

the portion of wealth $X_{t}$ invested at time $t$ in the $i$-th asset, then by (1.2) we find that the strategies we are interested in are $\mathcal{F}_{t}$-measurable processes such that $\left(\alpha_{t}, \pi_{t}\right) \in \mathcal{A}\left(\theta_{t}\right)$ a.s., where

$$
\mathcal{A}(\theta)=\left\{(\alpha, \pi) \in[0,1] \times \Delta_{0}: \alpha+\frac{1}{\theta \cdot \mathbf{1}} c(\theta,(\theta \cdot \mathbf{1}) \pi)+\pi \cdot \mathbf{1}=1\right\} .
$$

At time $t+1$, due to securities price changes, the investor's portfolio changes to

$$
\theta_{t+1, i}=\theta_{t, i}^{+} \zeta_{t, i}=X_{t} \pi_{t, i} \zeta_{t, i}=h_{i}\left(\pi_{t}, \zeta_{t}, \theta_{t}\right), \quad i=1, \ldots, d,
$$

Equation (1.4) describes dynamics of a control system we are dealing with: $\theta_{t} \in[0, \infty)^{d}$ is regarded as a state of this system, $\left(\alpha_{t}, \pi_{t}\right) \in[0,1] \times \Delta_{0}$ are its control parameters, constrained by (1.3) describing admissible strategies, and the initial condition is given by portfolio $\theta=\theta_{0}$.

1.2. Investors and utility functions. We consider a sequence of investors with preferences described by strictly increasing and strictly concave utility functions $U_{t}^{n}:(0, \infty) \rightarrow \mathbb{R}$, $0 \leq t \leq T, n \in \overline{\mathbb{N}}$. Since we would like to study the behaviour of optimal strategies when investor preferences converge, we make the following:

Assumption 1.1. For all $t=0, \ldots, T$ and $x \in(0, \infty)$ we have

$$
U_{t}^{n}(x) \rightarrow U_{t}^{\infty}(x) \quad \text { if } n \rightarrow \infty .
$$

An investor, whose preferences are measured by functions $U_{t}^{\infty}$ will be sometimes called a "limit investor". Investors want to maximize expected utility from consumption 
and terminal wealth, precisely the following reward functional:

$$
J_{T}^{n}(\theta,(\alpha, \pi))=\mathbb{E}\left(\sum_{t=0}^{T-1} U_{t}^{n}\left(\alpha_{t} \theta_{t} \cdot \mathbf{1}\right)+U_{T}^{n}\left(\theta_{T} \cdot \mathbf{1}\right)\right) .
$$

REMARK 1.2. One can consume all or nothing of one's wealth, thus we need values of utility functions at 0 . We deal with that problem by putting $U(0):=\lim _{x \rightarrow 0^{+}} U(x)$; if this limit is finite, continuity and concavity properties hold, and if not (as with the logarithmic function), it is clear that the investor will not choose such a strategy when maximizing utility.

For a dynamic programming problem to be well posed and finite, we assume that the following conditions are satisfied:

Assumption 1.3. For all $n \in \overline{\mathbb{N}}, k \in\{1, \ldots, T\}$ and $x>0$ we have

$$
\begin{aligned}
& \mathbb{E}\left(U_{k}^{n}\right)^{+}\left(x \prod_{t=0}^{k-1} \max \left\{\zeta_{t, i}: i=1, \ldots, d\right\}\right)<\infty, \\
& \mathbb{E}\left(U_{k}^{n}\right)^{-}\left(x \prod_{t=0}^{k-1} \min \left\{\zeta_{t, i}: i=1, \ldots, d\right\}\right)<\infty .
\end{aligned}
$$

1.3. Transaction costs. The main point of our interest is the model with proportional transaction costs, that is, with the cost function $c:[0, \infty)^{d} \times[0, \infty)^{d} \rightarrow \mathbb{R}$ defined as

$$
c(x, y):=\sum_{i=1}^{d} c_{i}^{1}\left(x_{i}-y_{i}\right)^{+}+\sum_{i=1}^{d} c_{i}^{2}\left(x_{i}-y_{i}\right)^{-}, \quad x, y \in[0, \infty)^{d},
$$

with fixed constants $0<c_{i}^{j}<1, j=1,2, i=1, \ldots, d$. In the case $c_{i}^{1}=c_{i}^{2}=\gamma, i=1, \ldots, d$, we have the following simple form:

$$
c(x, y)=\gamma \sum_{i=1}^{d}\left|x_{i}-y_{i}\right|, \quad x, y \in[0, \infty)^{d} .
$$

Let us note that for proportional costs, because of the identity

$$
c(\lambda x, \lambda y)=\lambda c(x, y), \quad \lambda>0, x, y \in[0, \infty)^{d},
$$

we can describe admissible strategies as those $\mathcal{F}_{t}$-measurable random variables $\left(\alpha_{t}, \pi_{t}\right) \in$ $[0,1] \times \Delta_{0}$ a.s. for which

$$
\alpha_{t}+c\left(\theta_{t} /\left(\theta_{t} \cdot \mathbf{1}\right), \pi_{t}\right)+\pi_{t} \cdot \mathbf{1}=1 .
$$

On the real financial markets strictly convex transaction costs appear very rarely, recently they were used in [2] to model the liquidity effects. Here we will find them convenient for optimization, and use to approximate the proportional cost function. From now on, when talking about strictly convex costs we will understand the model with the cost function $c:[0, \infty)^{d} \times[0, \infty)^{d} \rightarrow \mathbb{R}$ which is strictly convex, that is:

$$
c\left(\lambda\left(x_{1}, y_{1}\right)+(1-\lambda)\left(x_{2}, y_{2}\right)\right)<\lambda c\left(x_{1}, y_{1}\right)+(1-\lambda) c\left(x_{2}, y_{2}\right),
$$

for any $\lambda \in(0,1), x_{1}, x_{2}, y_{1}, y_{2} \in[0, \infty)^{d},\left(x_{1}, y_{1}\right) \neq\left(x_{2}, y_{2}\right)$.

If we fix a proportional cost function $c$, it is easy to construct an increasing sequence $c_{k}$ of strictly convex cost functions convergent to $c$. To every cost function of this sequence in 
a natural way there corresponds a set of admissible strategies. Namely, when we denote by $\mathcal{A}^{k}(\theta)$ the set $\mathcal{A}(\theta)$ defined in (1.3) with $c$ replaced by $c_{k}$, then the set $\mathcal{A}_{t}^{k}(\theta)$ of strategies admissible at time $t$ for an investor with portfolio $\theta$, at the market with cost function $c_{k}$, consists of $\mathcal{F}_{t}$-measurable random variables such that $(\alpha, \pi) \in \mathcal{A}^{k}(\theta)$ a.s. We also denote by $\mathcal{A}^{\infty}(\theta)$ and $\mathcal{A}_{t}^{\infty}(\theta)$ analogous sets for proportional costs. If we set $\overline{\mathcal{A}}^{k}(\theta)=\operatorname{conv}\left(\mathcal{A}^{k}(\theta) \cup\{0\}\right), k \in \overline{\mathbb{N}}$, where $\operatorname{conv}(A)$ denotes the convex hull of the set $A$, we see that the sets $\overline{\mathcal{A}}^{k}(\theta)$ forms a decreasing chain of closed subsets of the set $[0,1] \times \Delta_{0}$, and moreover

$$
\bigcap_{k=1}^{\infty} \overline{\mathcal{A}}^{k}(\theta)=\overline{\mathcal{A}}^{\infty}(\theta)
$$

2. One step case. We start with the case $T=1$. We assume that $\mathcal{H}$ is a sub- $\sigma$ field of $\mathcal{F}$, and denote by $\mathbb{E}(\cdot \mid \mathcal{H})$ the conditional expectation with respect to $\mathcal{H}$. Let the $\mathcal{H}$-measurable and $\mathbb{R}_{++}^{d}$-valued r.v. $\theta$ be an initial portfolio and $\zeta=\left(\zeta_{1}, \ldots, \zeta_{d}\right)$ be a $d$-dimensional r.v. with almost surely strictly positive coordinates, which corresponds to the relative change of share prices (cf. (1.1)). By $\kappa$ we denote the regular conditional distribution of $\zeta$ given $\mathcal{H}$ (it exists by [6, Theorem 2.7.5]).

We fix a strictly convex cost function $c$, and recall that the set of strategies admissible from portfolio $\theta$ consists of $\mathcal{H}$-measurable random variables $(\alpha, \pi) \in \mathcal{A}(\theta)$ a.s. By abuse of notation we denote this set by $\mathcal{A}(\theta)$ also. In the sequel let $\Theta$ denote the class of strictly concave and strictly increasing functions $u:(0, \infty) \rightarrow \mathbb{R}$, and let $\Xi$ denote the class of functions $v: \mathbb{R}_{++}^{d} \times \Omega \rightarrow \mathbb{R}$ such that

i) $v(\cdot, \omega)$ is continuous, strictly concave and strictly increasing with respect to every coordinate for almost all $\omega$,

ii) $v(\theta, \cdot)$ is $\mathcal{F}$-measurable for $\theta \in \mathbb{R}_{++}^{d}$.

Let us fix functions $u \in \Theta, v \in \Xi$ and define value function $w: \mathbb{R}_{++}^{d} \times \Omega \rightarrow \mathbb{R}$ (from now on, to simplify notation, we suppress dependency on $\omega$ where it is possible):

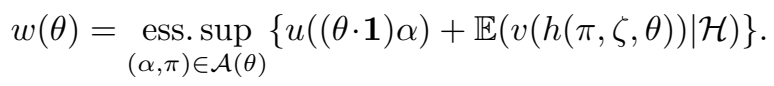

Analogously to Assumption 1.3, we impose

Assumption 2.1. For all $x>0$ and $\pi \in \Delta_{0}$ assume

$$
\mathbb{E} v^{+}\left(x \max \left\{\zeta_{i}: i=1, \ldots, d\right\}\right)<\infty, \quad \mathbb{E} v^{-}\left(x \min \left\{\zeta_{i}: i=1, \ldots, d\right\}\right)<\infty .
$$

We have the following technical lemma.

LEMma 2.2. If $v \in \Xi$, then for every $\pi \in \Delta_{0}$ there exists a version of the mapping

$$
\mathbb{R}_{++}^{d} \ni \theta \mapsto \mathbb{E}(v(h(\pi, \zeta, \theta)) \mid \mathcal{H})
$$

that belongs to the class $\Xi$.

Proof. By routine calculations one checks that the version defined by

$$
\mathbb{E}(v(h(\pi, \zeta, \theta)) \mid \mathcal{H})(\omega)=\int_{\mathbb{R}^{d}} v(h(\pi, x, \theta)) \kappa(d x, \omega)
$$

has the desired properties. 
The most important step is to prove existence and uniqueness of optimal strategies. We obtain existence due to compactness of the sets $\mathcal{A}(\theta(\omega))$. Uniqueness is a consequence of strict concavity of the utility function and strict convexity of the cost function.

Proposition 2.3. For every initial portfolio $\theta \in \mathbb{R}_{++}^{d}$, there exists a unique optimal strategy $(\hat{\alpha}, \hat{\pi}) \in \mathcal{A}(\theta)$ such that

$$
w(\theta)=u((\theta \cdot \mathbf{1}) \hat{\alpha})+\mathbb{E}(v(h(\hat{\pi}, \zeta, \theta)) \mid \mathcal{H}), \text { a.s. }
$$

Proof. Fix $\theta \in \mathbb{R}_{++}^{d}$ and take a version of conditional expectation with the properties stated in Lemma 2.2. Let us denote by $\Omega^{\prime}$ the set on which conditional expectation is continuous. Then the mapping $\Phi: \mathcal{A}(\theta) \times \Omega \rightarrow[-\infty, \infty)$ defined as

$$
\Phi(\alpha, \pi, \omega)= \begin{cases}u((\theta \cdot \mathbf{1}) \alpha)+\mathbb{E}(v(h(\pi, \zeta, \theta)) \mid \mathcal{H}), & \omega \in \Omega^{\prime}, \\ -\infty, & \text { otherwise }\end{cases}
$$

is $\mathcal{B}\left([0,1] \times \Delta_{0}\right) \otimes \mathcal{H}$-measurable and continuous for all $\omega$. Since the set $\mathcal{A}(\theta)$ is compact, also the sets $\{(\alpha, \pi): \Phi(\alpha, \pi, \omega) \geq c\}$ are compact for all $c \in \mathbb{R}$ and $\omega$. Thus, by Lemmas 3 and 4 in [3], there exists an $\mathcal{H}$-measurable pair $(\hat{\alpha}(\omega), \hat{\pi}(\omega))$ that attains the supremum of $\Phi$.

Suppose that there are two such pairs, say $\left(\alpha^{1}, \pi^{1}\right),\left(\alpha^{2}, \pi^{2}\right) \in \mathcal{A}(\theta)$. For $\lambda \in(0,1)$, let $\alpha=\lambda \alpha^{1}+(1-\lambda) \alpha^{2}$ and $\pi=\lambda \pi^{1}+(1-\lambda) \pi^{2}$. Clearly $\alpha \in(0,1)$ and $\pi \in \Delta_{0}$ a.s. From the strict convexity of $c$ we have

$$
c((\theta \cdot \mathbf{1}) \pi, \theta)<\lambda c\left((\theta \cdot \mathbf{1}) \pi^{1}, \theta\right)+(1-\lambda) c\left((\theta \cdot \mathbf{1}) \pi^{2}, \theta\right),
$$

hence $\pi \cdot \mathbf{1}<1-\alpha-\frac{1}{\theta \cdot \mathbf{1}} c((\theta \cdot \mathbf{1}) \pi, \theta)$, and there exists an $\mathcal{H}$-measurable r.v. $\varepsilon \geq 1$ such that $(\alpha, \varepsilon \pi) \in \mathcal{A}(\theta)$. Thus from the strict convexity of $v$ :

$$
\begin{aligned}
w(\theta)= & \lambda w(\theta)+(1-\lambda) w(\theta) \\
= & \lambda\left[u\left((\theta \cdot \mathbf{1}) \alpha^{1}\right)+\mathbb{E}\left(v\left(h\left(\pi^{1}, \zeta, \theta\right)\right) \mid \mathcal{H}\right)\right] \\
& \quad+(1-\lambda)\left[u\left((\theta \cdot \mathbf{1}) \alpha^{2}\right)+\mathbb{E}\left(v\left(h\left(\pi^{2}, \zeta, \theta\right)\right) \mid \mathcal{H}\right)\right] \\
& <u((\theta \cdot \mathbf{1}) \alpha)+\mathbb{E}(v(h(\varepsilon \pi, \zeta, \theta)) \mid \mathcal{H}) \leq w(\theta) .
\end{aligned}
$$

This contradiction proves the uniqueness of optimal strategy.

To use the dynamic programming method, we must ensure regularity of the value functions.

LEMMA 2.4. For $\theta \in \mathbb{R}_{++}^{d}$ and $\omega \in \Omega$ define the version of value function

$$
w(\theta, \omega)=u((\theta \cdot \mathbf{1}) \hat{\alpha}(\theta, \omega))+\int_{\mathbb{R}^{d}} v(h(\hat{\pi}(\theta, \omega), x, \theta)) \kappa(d x, \omega),
$$

where $(\hat{\alpha}, \hat{\pi})$ is the optimal control for $\theta$. This version belongs to the class $\Xi$.

We skip the proof consisting of standard calculations. Another fact will be also useful.

Proposition 2.5. There exists a selector of optimal strategies

$$
\mathbb{R}_{++}^{d} \ni \theta \mapsto(\hat{\alpha}, \hat{\pi})(\theta) \in \mathcal{A}(\theta)
$$

continuous for almost all $\omega$. 
Proof. Let us fix a continuous version of conditional expectation (cf. Lemma 2.2). Then the random function

$$
w(\theta,(\alpha, \pi))=u((\theta \cdot \mathbf{1}) \alpha)+\mathbb{E}(v(h(\pi, \zeta, \theta)) \mid \mathcal{H})
$$

is also continuous (jointly for all variables) off a $P$-null set $N$. Fix $\omega \in \Omega \backslash N$ and suppose there exist $\theta \in \mathbb{R}_{++}^{d}$ and a sequence $\left(\theta^{n} \in \mathbb{R}_{++}^{d}: n \in \mathbb{N}\right)$ such that $\theta^{n} \rightarrow \theta$, but $(\hat{\alpha}, \hat{\pi})\left(\theta^{n}, \omega\right) \nrightarrow(\hat{\alpha}, \hat{\pi})(\theta, \omega)$. Since $(\hat{\alpha}, \hat{\pi})\left(\theta^{n}, \omega\right) \in[0,1] \times \Delta_{0}$ for $n \in \mathbb{N}$, we can choose a subsequence $(\hat{\alpha}, \hat{\pi})\left(\theta^{n_{k}}, \omega\right)$ convergent to $(\tilde{\alpha}, \tilde{\pi}) \in[0,1] \times \Delta_{0}$. Moreover, since the condition

$$
\hat{\alpha}\left(\theta^{n_{k}}\right)+\hat{\pi}\left(\theta^{n_{k}}\right) \cdot \mathbf{1}+\frac{1}{\theta^{n_{k}} \cdot \mathbf{1}} c\left(\left(\theta^{n_{k}} \cdot \mathbf{1}\right) \hat{\pi}\left(\theta^{n_{k}}\right), \theta^{n_{k}}\right)=1
$$

is fulfilled for all $k$, we conclude that $(\tilde{\alpha}, \tilde{\pi}) \in \mathcal{A}(\theta)$. By continuity

$$
\lim _{k \rightarrow \infty} w\left(\theta^{n_{k}},(\hat{\alpha}, \hat{\pi})\left(\theta^{n_{k}}, \omega\right)\right)=w(\theta,(\tilde{\alpha}, \tilde{\pi}))=: \tilde{w}
$$

and

$$
\lim _{n \rightarrow \infty} w\left(\theta^{n},(\hat{\alpha}, \hat{\pi})(\theta, \omega)\right)=w(\theta,(\hat{\alpha}, \hat{\pi})(\theta, \omega))=: w .
$$

Since $(\tilde{\alpha}, \tilde{\pi}) \neq(\hat{\alpha}, \hat{\pi})(\theta, \omega)$, then $\tilde{w}<w$, hence if we fix $\varepsilon \in\left(0, \frac{w-\tilde{w}}{2}\right)$, then for large enough $k$ we have

$$
w\left(\theta^{n_{k}},(\hat{\alpha}, \hat{\pi})(\theta, \omega)\right)>w-\varepsilon>\tilde{w}+\varepsilon>w\left(\theta^{n_{k}},(\hat{\alpha}, \hat{\pi})\left(\theta^{n_{k}}, \omega\right)\right)
$$

contradicting optimality of $(\hat{\alpha}, \hat{\pi})\left(\theta^{n_{k}}, \omega\right)$.

We are now ready to prove the convergence of optimal strategies for $T=1$.

Proposition 2.6. Assume that functions $u_{n} \in \Theta, v_{n} \in \Xi, n \in \overline{\mathbb{N}}$, are such that $\lim _{n \rightarrow \infty} u_{n}=u_{\infty}$ and $\lim _{n \rightarrow \infty} v_{n}=v_{\infty}$, and let $\left(\hat{\alpha}_{n}, \hat{\pi}_{n}\right)$ be the optimal strategies fulfilling (2.1) with $u$ and $v$ replaced respectively with $u_{n}$ and $v_{n}$. Then

$$
\lim _{n \rightarrow \infty}\left(\hat{\alpha}_{n}, \hat{\pi}_{n}\right)(\theta)=\left(\hat{\alpha}_{\infty}, \hat{\pi}_{\infty}\right)(\theta) \text {, a.s., } \theta \in \mathbb{R}_{++}^{d} .
$$

Proof. Suppose on the contrary that the convergence does not hold for some $\theta \in \mathbb{R}_{++}^{d}$. Since $[0,1] \times \Delta_{0}$ is a compact set, by Lemma (A.1) we may choose a random subsequence $\left(n_{k} \in \mathbb{N}: k \in \mathbb{N}\right)$ such that $\lim _{k \rightarrow \infty}\left(\hat{\alpha}_{n_{k}}, \hat{\pi}_{n_{k}}\right)(\theta)=(\tilde{\alpha}, \tilde{\pi}) \in \mathcal{A}(\theta)$ a.s., $(\tilde{\alpha}, \tilde{\pi}) \neq\left(\hat{\alpha}_{\infty}, \hat{\pi}_{\infty}\right)$. Define, for $n \in \overline{\mathbb{N}}$, functions

$$
w_{n}(\alpha, \pi)=u_{n}((\theta \cdot \mathbf{1}) \alpha)+\mathbb{E}\left(v_{n}(h(\pi, \zeta, \theta)) \mid \mathcal{H}\right),(\alpha, \pi) \in \mathcal{A}(\theta),
$$

with continuous version of conditional expectation (cf. Lemma 2.2). The functions $w_{n}$ are almost surely continuous, hence from almost uniform convergence of the sequences $u_{n}$ and $v_{n}$ (cf. Lemma A.2)

$$
\lim _{k \rightarrow \infty} w_{n_{k}}\left(\hat{\alpha}_{n_{k}}, \hat{\pi}_{n_{k}}\right)=w_{\infty}(\tilde{\alpha}, \tilde{\pi}) \text {, a.s. }
$$

and since $(\tilde{\alpha}, \tilde{\pi})$ is not optimal for $w_{\infty}$, we have

$$
\tilde{w}:=w_{\infty}(\tilde{\alpha}, \tilde{\pi})<w_{\infty}\left(\hat{\alpha}_{\infty}, \hat{\pi}_{\infty}\right)=\lim _{k \rightarrow \infty} w_{n_{k}}\left(\hat{\alpha}_{\infty}, \hat{\pi}_{\infty}\right)=: w \text {, a.s. }
$$

Hence, if $\varepsilon$ is such that $0<\varepsilon<\frac{w-\tilde{w}}{2}$ a.s., then for large enough $k$ :

$$
w_{n_{k}}\left(\hat{\alpha}_{\infty}, \hat{\pi}_{\infty}\right)>w-\varepsilon>\tilde{w}+\varepsilon>w_{n_{k}}\left(\hat{\alpha}_{n_{k}}, \hat{\pi}_{n_{k}}\right),
$$

which contradicts optimality of $\left(\hat{\alpha}_{n_{k}}, \hat{\pi}_{n_{k}}\right)$. 
3. Main results. Now we can deal with the case of general $T$, and present the main results of the paper.

For every $k \in \overline{\mathbb{N}}$ and $n \in \overline{\mathbb{N}}$ we define the Bellman functions:

$$
\begin{aligned}
& V_{n, T}^{k}(\theta)=U_{n, T}(\theta \cdot \mathbf{1}),
\end{aligned}
$$

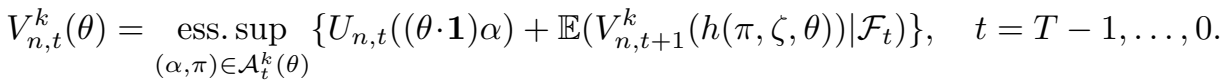

Let us recall that the superscript $k$ denotes $k$-th strictly convex cost function in the sequence approximating the proportional cost function $c$ (which corresponds to $k=\infty$ ), and the subscript $n$ belongs to the $n$-th investor and its preferences expressed by the utility functions $U_{n, t}$.

For the strictly convex costs case we have the following:

Proposition 3.1. For $k \in \mathbb{N}, n \in \overline{\mathbb{N}}$ and $t=0, \ldots, T-1$, the functions $V_{n, t}^{k}$ have versions which are almost surely strictly concave and strictly increasing for each variable. Moreover, there exist unique $\mathcal{B}\left(\mathbb{R}_{++}^{d}\right) \otimes \mathcal{F}_{t}$-measurable functions $\left(\hat{\alpha}_{n, t}^{k}, \hat{\pi}_{n, t}^{k}\right) \in \mathcal{A}_{t}^{k}(\cdot)$ such that for all $\theta \in \mathbb{R}_{++}^{d}$ we have:

$$
V_{n, t}^{k}(\theta)=U_{n, t}\left(\hat{\alpha}_{n, t}^{k}(\theta) \theta \cdot \mathbf{1}\right)+\mathbb{E}\left(V_{n, t+1}^{k}\left(h\left(\hat{\pi}_{n, t}^{k}(\theta), \zeta_{t}, \theta\right)\right) \mid \mathcal{F}_{t}\right), \text { a.s. }
$$

Proof. Fix $k \in \mathbb{N}, n \in \overline{\mathbb{N}}$, and use backward induction in $t$. It is trivial that $V_{n, T}^{k} \in \Xi$, since $U_{n, T} \in \Theta$. Letting $t$ decrease from $T-1$ to 0 , we set $w=V_{n, t}^{k}, u=U_{n, t}, v=V_{n, t+1}^{k}, \mathcal{H}=\mathcal{F}_{t}$ and $\zeta=\zeta_{t}$. Using Lemma 2.3 we obtain a unique optimal strategy $\left(\hat{\alpha}_{n, t}^{k}, \hat{\pi}_{n, t}^{k}\right)=(\hat{\alpha}, \hat{\pi})$, $\mathcal{F}_{t}$-measurable for all $\theta \in \mathbb{R}_{++}^{d}$, and almost surely continuous by Lemma 2.5 , hence $\mathcal{B}\left(\mathbb{R}_{++}^{d}\right) \otimes \mathcal{F}_{t}$-measurable (cf. [4, Theorem 6.1]). Lemma 2.4 guarantees that $V_{n, t}^{k} \in \Xi$, which by induction completes the proof.

Having proved existence and uniqueness of optimal strategies, we are able to prove their convergence. Again we make use of the results from the one-step case and backward induction.

Proposition 3.2. Let $k \in \mathbb{N}$ and $\left(\left(\hat{\alpha}_{n, t}^{k}, \hat{\pi}_{n, t}^{k}\right): t=0, \ldots, T-1\right), n \in \overline{\mathbb{N}}$, be optimal strategies for investors maximizing reward functional (1.5) on the market with strictly convex transaction cost function $c_{k}$. Then

$$
\lim _{n \rightarrow \infty}\left(\hat{\alpha}_{n, t}^{k}, \hat{\pi}_{n, t}^{k}\right)(\theta)=\left(\hat{\alpha}_{\infty, t}^{k}, \hat{\pi}_{\infty, t}^{k}\right)(\theta) \quad \text { and } \quad \lim _{n \rightarrow \infty} V_{n, t}^{k}(\theta)=V_{\infty, t}^{k}(\theta) \text {, a.s. }
$$

for $\theta \in \mathbb{R}_{++}^{d}$ and $t=0, \ldots, T-1$.

Proof. We use Proposition 2.6 to the functions $u_{n}=U_{n, t}$ and $v_{n}=V_{n, t+1}^{k}$, in sequence for $t=T-1, \ldots, 0$. We need to check that $\lim _{n \rightarrow \infty} V_{n, t}^{k}(\theta)=V_{\infty, t}^{k}(\theta)$. For $t=T$ this is trivial, and if we have shown that $\left(\hat{\alpha}_{n, t}^{k}, \hat{\pi}_{n, t}^{k}\right) \rightarrow\left(\hat{\alpha}_{\infty, t}^{k}, \hat{\pi}_{\infty, t}^{k}\right)$ for given $t$, then uniform convergence on compact sets and the Lebesgue Theorem for conditional expectations give us

$$
\begin{aligned}
\lim _{n \rightarrow \infty} V_{n, t}^{k}(\theta) & =\lim _{n \rightarrow \infty}\left(U_{n, t}\left((\theta \cdot \mathbf{1}) \hat{\alpha}_{n, t}^{k}(\theta)\right)+\mathbb{E}\left(V_{n, t+1}^{k}\left(h\left(\hat{\pi}_{n, t}^{k}(\theta), \zeta_{t}, \theta\right)\right) \mid \mathcal{F}_{t}\right)\right) \\
& =U_{\infty, t}\left((\theta \cdot \mathbf{1}) \hat{\alpha}_{\infty, t}^{k}(\theta)\right)+\mathbb{E}\left(V_{\infty, t+1}^{k}\left(h\left(\hat{\pi}_{\infty, t}^{k}(\theta), \zeta_{t}, \theta\right)\right) \mid \mathcal{F}_{t}\right) \\
& =V_{\infty, t}^{k}(\theta), \text { a.s., } \theta \in \mathbb{R}_{++}^{d} .
\end{aligned}
$$


Approximating proportional costs by strictly convex costs we are now going to obtain existence of optimal strategies for proportional cost markets. Moreover, it turns out that these strategies may be approximated by subsequences of optimal strategies for strictly convex costs. In the same way we are able to approximate value functions.

THEOREM 3.3. For every $n \in \overline{\mathbb{N}}$ there exist almost surely continuous and concave version of Bellman functions $V_{n, t}^{\infty}$ and optimal strategies $\left(\hat{\alpha}_{n, t}^{\infty}, \hat{\pi}_{n, t}^{\infty}\right)$ for the problem (1.5) with proportional transaction costs. Moreover, there exists a random subsequence $\left(k_{l} \in \mathbb{N}\right.$ : $l \in \mathbb{N})$ such that for $t=0, \ldots, T-1$ :

$$
\lim _{l \rightarrow \infty}\left(\hat{\alpha}_{n, t}^{k_{l}}, \hat{\pi}_{n, t}^{k_{l}}\right)=\left(\hat{\alpha}_{n, t}^{\infty}, \hat{\pi}_{n, t}^{\infty}\right) \quad \text { and } \quad \lim _{l \rightarrow \infty} V_{n, t}^{k_{l}}=V_{n, t}^{\infty} \text {, a.s. }
$$

Proof. Backward induction in $t=T, \ldots, 0$. For $t=T$, we have that $V_{n, T}^{k}(\theta)=U_{n, T}(\theta \cdot \mathbf{1})$ for every $k \in \overline{\mathbb{N}}$, hence it is continuous and concave. For $t<T$, notice that the sequence $\left(V_{n, t}^{k}: k \in \mathbb{N}\right)$ is decreasing and bounded from below by $V_{n, t}^{\infty}$, hence convergent to a function $\tilde{V}_{n, t}$. For every fixed $\theta \in \mathbb{R}_{++}^{d}$, by Lemma A.1 we can choose a subsequence $k_{l}=$ $k_{l}(t, \theta)$, such that $\left(\hat{\alpha}_{n, t}^{k_{l}}, \hat{\pi}_{n, t}^{k_{l}}\right)(\theta)$ converges, and by (1.6) its limit denoted $\left(\hat{\alpha}_{n, t}^{\infty}, \hat{\pi}_{n, t}^{\infty}\right)(\theta)$ belongs to $\mathcal{A}^{\infty}(\theta)$. Thus

$$
\begin{aligned}
V_{n, t}^{\infty}(\theta) & \leq \tilde{V}_{n, t}(\theta)=\lim _{k \rightarrow \infty} V_{n, t}^{k}(\theta)=\lim _{l \rightarrow \infty} V_{n, t}^{k_{l}}(\theta) \\
& =\lim _{l \rightarrow \infty}\left(U_{n, t}\left((\theta \cdot \mathbf{1}) \hat{\alpha}_{n, t}^{k_{l}}(\theta)\right)+\mathbb{E}\left(V_{n, t+1}^{k_{l}}\left(h\left(\hat{\pi}_{n, t}^{k_{l}}(\theta), \zeta_{t}, \theta\right)\right) \mid \mathcal{F}_{t}\right)\right) \\
& =U_{n, t}\left((\theta \cdot \mathbf{1}) \hat{\alpha}_{n, t}^{\infty}(\theta)\right)+\mathbb{E}\left(V_{n, t+1}^{\infty}\left(h\left(\hat{\pi}_{n, t}^{\infty}(\theta), \zeta_{t}, \theta\right)\right) \mid \mathcal{F}_{t}\right) \leq V_{n, t}^{\infty}(\theta),
\end{aligned}
$$

where the last equality is justified by almost uniform convergence. Therefore the last inequality above is an equality, hence $V_{n, t}^{\infty}(\theta)=\lim _{k \rightarrow \infty} V_{n, t}^{k}(\theta)$ has an almost surely continuous version, which is also concave as a limit of concave functions and the strategy $\left(\hat{\alpha}_{n, t}^{\infty}, \hat{\pi}_{n, t}^{\infty}\right)(\theta)$ is optimal for $k=\infty$.

Unfortunately we are not able to ensure the uniqueness of optimal strategies here, hence the method used to prove the convergence of optimal strategies with respect to preferences on the market with strictly convex costs doesn't work with proportional ones. However, we have the convergence of value functions, and in the proportional cost analogue of Proposition 3.2 we pass to the subsequences.

TheOREM 3.4. For every $t=0, \ldots, T$ and $\theta \in \mathbb{R}_{++}^{d}$ we have

$$
\lim _{n \rightarrow \infty} V_{n, t}^{\infty}(\theta)=V_{\infty, t}^{\infty}(\theta), \text { a.s. }
$$

Proof. Fix $\theta \in \mathbb{R}_{++}^{d}$. Consecutively for $t=T-1, \ldots, 0$ we have

$$
V_{n, t}^{\infty}(\theta) \geq U_{n, t}\left((\theta \cdot \mathbf{1}) \hat{\alpha}_{\infty, t}^{\infty}\right)+\mathbb{E}\left(V_{n, t+1}^{\infty}\left(h\left(\hat{\pi}_{\infty, t}^{\infty}, \zeta_{t}, \theta\right)\right) \mid \mathcal{F}_{t}\right)
$$

for $n \in \mathbb{N}$, and hence

$$
\liminf _{n \rightarrow \infty} V_{n, t}^{\infty}(\theta) \geq V_{\infty, t}^{\infty}(\theta) .
$$

On the other hand, if we $\operatorname{suppose}$ that $\limsup _{n \rightarrow \infty} V_{n, t}^{\infty}(\theta)>V_{\infty, t}^{\infty}(\theta)$, then there exist $\delta>0$ and a subsequence $\left(n_{l}: l \in \mathbb{N}\right)$ such that $V_{n_{l}, t}^{\infty}(\theta)>V_{\infty, t}^{\infty}(\theta)+\delta$. For every $l$ we can choose an index $k_{l}>k_{l-1}$ such that $V_{n_{l}, t}^{k_{l}}(\theta)>V_{n_{l}, t}^{\infty}(\theta)-\delta / 2$, hence $V_{n_{l}, t}^{k_{l}}(\theta)>$ 
$V_{\infty, t}^{\infty}(\theta)+\delta / 2$. From the sequence $\left(\hat{\alpha}_{n_{l}, t}^{k_{l}}, \hat{\pi}_{n_{l}, t}^{k_{l}}\right)$ we can choose a (random) subsequence convergent to $(\hat{\alpha}, \hat{\pi}) \in \mathcal{A}^{\infty}(\theta)$ and obtain

$$
\left.U_{\infty, t}((\theta \cdot \mathbf{1}) \hat{\alpha})+\mathbb{E}\left(V_{\infty, t+1}^{\infty}\left(h\left(\hat{\pi}, \zeta_{t}, \theta\right)\right) \mid \mathcal{F}_{t}\right)\right) \geq V_{\infty, t}^{\infty}(\theta)+\delta / 2,
$$

which is impossible. Thus $V_{\infty, t}^{\infty}(\theta) \geq \lim \sup _{n \rightarrow \infty} V_{n, t}^{\infty}(\theta)$.

Remark 3.5. Due to Theorem 3.3 and Proposition 3.2, equation (3.1) can be written in the following form:

$$
\lim _{n \rightarrow \infty} \lim _{k \rightarrow \infty} V_{n, t}^{k}(\theta)=\lim _{k \rightarrow \infty} \lim _{n \rightarrow \infty} V_{n, t}^{k}(\theta) \text {, a.s. }
$$

THEOREM 3.6. There exists a random subsequence $\left(n_{l}: l \in \mathbb{N}\right)$ such that if $\left(\hat{\alpha}_{n, t}^{\infty}, \hat{\pi}_{n, t}^{\infty}\right)$, $n \in \overline{\mathbb{N}}$, are optimal strategies for the problem (1.5) with proportional costs, then

$$
\lim _{l \rightarrow \infty}\left(\hat{\alpha}_{n_{l}, t}^{\infty}, \hat{\pi}_{n_{l}, t}^{\infty}\right)=\left(\hat{\alpha}_{\infty, t}^{\infty}, \hat{\pi}_{\infty, t}^{\infty}\right), \text { a.s. }
$$

Proof. For fixed $t=T-1, \ldots, 0$ and $\theta \in \mathbb{R}_{++}^{d}$, from the sequence $\left(\alpha_{n_{t}}^{\infty}, \pi_{n, t}^{\infty}\right)(\theta)$ of optimal strategies we may choose a (random) subsequence $\left(\alpha_{n_{k}, t}^{\infty}, \pi_{n_{k}, t}^{\infty}\right)$ convergent to some strategy $(\hat{\alpha}, \hat{\pi}) \in \mathcal{A}^{\infty}(\theta)$. Then

$$
\begin{aligned}
V_{\infty, t}^{\infty}(\theta) & =\lim _{k \rightarrow \infty} V_{n_{k}, t}^{\infty}(\theta) \\
& =\lim _{k \rightarrow \infty} U_{n_{k}, t}\left((\theta \cdot \mathbf{1}) \alpha_{n_{k}, t}^{\infty}\right)+\mathbb{E}\left(V_{n_{k}, t+1}^{\infty}\left(h\left(\pi_{n_{k}, t}^{\infty}, \zeta_{t}, \theta\right)\right) \mid \mathcal{F}_{t}\right) \\
& =U_{\infty, t}((\theta \cdot \mathbf{1}) \hat{\alpha})+\mathbb{E}\left(V_{\infty, t+1}^{\infty}\left(h\left(\hat{\pi}, \zeta_{t}, \theta\right)\right) \mid \mathcal{F}_{t}\right),
\end{aligned}
$$

hence the strategy $(\hat{\alpha}, \hat{\pi})$ is optimal for an investor with proportional transaction costs.

It is worth to remark that the above proof shows that if a sequence $\left(\hat{\alpha}_{n_{l}, t}^{\infty}, \hat{\pi}_{n_{l}, t}^{\infty}\right)$ of optimal strategies converges, then its limit is an optimal strategy for a "limit" investor.

\section{A. Appendix}

Lemma A.1 (Kabanov, Stricker). Let $\eta_{n} \in L^{0}\left(\mathbb{R}^{d}\right)$ be such that $\bar{\eta}:=\liminf _{n}\left|\eta_{n}\right|<\infty$. Then there are $\tilde{\eta}_{k} \in L^{0}\left(\mathbb{R}^{d}\right)$ such that for all $\omega$ the sequence of $\tilde{\eta}_{k}(\omega)$ is a convergent subsequence of the sequence of $\eta_{n}(\omega)$.

LEMmA A.2. If $f_{n}: \mathbb{R}_{++}^{d} \rightarrow \mathbb{R}$ is a sequence of functions increasing with respect to every variable, pointwise convergent to the continuous function $f: \mathbb{R}_{++}^{d} \rightarrow \mathbb{R}$, then the sequence $f_{n}$ converges almost uniformly to $f$ (uniformly on compact subsets of $\mathbb{R}_{++}^{d}$ ).

Proof. Fix an $\varepsilon>0$ and a compact set $C \subset \mathbb{R}_{++}^{d}$. Without loss of generality we may assume that $C=\prod_{i=1}^{d}\left[a_{i}, b_{i}\right]$ for some real numbers $a_{i} \leq b_{i}$. Since $f$ is uniformly continuous on $C$, we may find a finite sequence of points $x_{1}, \ldots, x_{k} \in C$ such that for any $x \in C$ there are $i, j \in\{1, \ldots, k\}$ with

$$
x_{i} \leq x \leq x_{j} \quad \text { oraz } f\left(x_{j}\right)-f\left(x_{i}\right)<\varepsilon / 2 .
$$

Let $N_{i} \in \mathbb{N}$ be such that $\left|f_{n}\left(x_{i}\right)-f\left(x_{i}\right)\right|<\frac{\varepsilon}{2}$ for $n \geq N_{i}$, and set $N=\max \left\{N_{i}: i=\right.$ $0, \ldots, k\}$. Moreover $f$ is also increasing in every coordinate, thus for any point $x \in C$ 
with the choice of indices $i, j$ as in (A.1), for $n \geq N$ we have:

$$
\begin{aligned}
-\varepsilon & <f\left(x_{i}\right)-f\left(x_{j}\right)-\frac{\varepsilon}{2} \\
& <f_{n}\left(x_{i}\right)-f\left(x_{j}\right) \leq f_{n}(x)-f(x) \leq f_{n}\left(x_{j}\right)-f\left(x_{i}\right) \\
& <f\left(x_{j}\right)-f\left(x_{i}\right)+\frac{\varepsilon}{2}<\varepsilon .
\end{aligned}
$$

\section{References}

[1] L. Carassus and M. Rásonyi, Optimal strategies and utility-based prices converge when agents' preferences do, Math. Oper. Res. 32 (2007), 102-117.

[2] U. Çetin and L. C. G. Rogers, Modelling liquidity effects in discrete time, Math. Finance 17 (2007), 15-29.

[3] I. V. Evstigneev, Measurable selection and dynamic programming, Math. Oper. Res. 3 (1976), 267-272.

[4] C. J. Himmelberg, Measurable relations, Fundam. Math. 87 (1975), 53-72.

[5] Yu. M. Kabanov and Ch. Stricker, A teachers' note on no-arbitrage criteria, in: Séminaire de Probabilités XXXV, Lecture Notes in Mathematics 1755, 2004, 149-152.

[6] A. N. Shiryaev, Probability, Graduate Texts in Mathematics 95, Springer-Verlag, New York, 1995.

[7] R. Kucharski, Convergence of optimal strategies in a discrete time market with finite horizon, Appl. Math. (Warsaw)( 33 (2006), 85-93.

[8] K. Larsen, Continuity of utility-maximization with respect to preferences, preprint, Math. Finance (2008), to appear.

[9] E. Jouini and C. Napp, Convergence of utility functions and convergence of optimal strategies, Finance Stoch. 8 (2004), 133-144.

[10] R. T. Rockafellar, Convex Analysis, Princeton University Press, 1970.

[11] M. Rásonyi and E. Stettner, On utility maximization in discrete-time financial market models, Ann. Appl. Probab. 15 (2005), 1367-1395.

[12] J. Zabczyk, Chance and Decision: Stochastic Control in Discrete Time, Scuola Norm. Sup., Pisa, 1996. 
